\title{
Gonorrhoea treatment failure caused by a Neisseria gonorrhoeae strain with combined ceftriaxone and high- level azithromycin resistance, England, February 2018
}

David W Eyre ${ }^{1,2}$, Nicholas D Sanderson ${ }^{2}$, Emily Lord ${ }^{3}$, Natasha Regisford-Reimmer ${ }^{3}$, Kevin Chau ${ }^{2}$, Leanne Barker ${ }^{2}$, Markus

Morgan $^{3}$, Robert Newnham 3 , Daniel Golparian ${ }^{4}$, Magnus Unemo ${ }^{4}$, Derrick W Crook ${ }^{2,5,6}$, Tim EA Peto ${ }^{2,6}$, Gwenda Hughes ${ }^{5}$, Michelle J Cole ${ }^{5}$, Helen Fifer ${ }^{5}$, Anne Edwards ${ }^{3,7}$, Monique I Andersson ${ }^{3,7}$

1. Big Data Institute, University of Oxford, Oxford, United Kingdom

2. Nuffield Department of Medicine, University of Oxford, Oxford, United Kingdom

3. Oxford University Hospitals NHS Foundation Trust, Oxford, United Kingdom

4. WHO Collaborating Centre for Gonorrhoea and Other STIs, National Reference Laboratory for STIs, Örebro University Hospital, Örebro, Sweden

5. National Infection Service, Public Health England, Colindale, United Kingdom

6. National Institute for Health Research Health Protection Research Unit in Healthcare Associated Infections and Antimicrobial Resistance, University of Oxford, Oxford, United Kingdom

7. The authors contributed equally to this work

Correspondence: David Eyre (david.eyre@bdi.ox.ac.uk)

Citation style for this article:

Eyre David W, Sanderson Nicholas D, Lord Emily, Regisford-Reimmer Natasha, Chau Kevin, Barker Leanne, Morgan Markus, Newnham Robert, Golparian Daniel, Unemo Magnus, Crook Derrick W, Peto Tim EA, Hughes Gwenda, Cole Michelle J, Fifer Helen, Edwards Anne, Andersson Monique I. Gonorrhoea treatment failure caused by a Neisseria gonorrhoeae strain with combined ceftriaxone and high-level azithromycin resistance, England, February 2018 . Euro Surveill. 2018;23(27):pii=1800323. https://doi.org/10.2807/1560-7917.ES.2018.23.27.1800323

We describe a gonorrhoea case with combined highlevel azithromycin resistance and ceftriaxone resistance. In February 2018, a heterosexual male was diagnosed with gonorrhoea in the United Kingdom following sexual intercourse with a locally resident female in Thailand and failed treatment with ceftriaxone plus doxycycline and subsequently spectinomycin. Resistance arose from two mechanisms combining for the first time in a genetic background similar to a commonly circulating strain. Urgent action is essential to prevent further spread.

Antimicrobial resistance in Neisseria gonorrhoeae is a major concern. Dual therapy with ceftriaxone and azithromycin, the last two mainstream agents to which $N$. gonorrhoeae remains largely susceptible, is widely recommended internationally [1]. We describe a case of urethral and pharyngeal infection with $N$. gonorrhoeae with combined high-level azithromycin resistance and ceftriaxone resistance. Previously no such cases have been reported.

\section{Case description and microbiology}

In February 2018, a heterosexual male presented to a sexual health clinic in the United Kingdom (UK) with a 4-day history of urethral discharge and dysuria. He reported having had sexual intercourse 3 days earlier with a regular female partner in the UK. He also reported having had sex with a locally resident female in Thailand in January 2018; he had no history of sexually transmitted infections and no other past medical history. Examination revealed a creamy white urethral discharge, with $3+$ pus cells and Gram-negative intracellular diplococci seen under microscopy, leading to a diagnosis of urethral gonorrhoea infection. He was treated with a single dose of intramuscular ceftriaxone $1 \mathrm{~g}$ and oral doxycycline $100 \mathrm{mg}$ twice daily for 7 days.

A urine nucleic acid amplification test (NAAT) was positive for $N$. gonorrhoeae and Chlamydia trachomatis negative. N. gonorrhoeae was cultured from a urethral swab. Antimicrobial susceptibility testing was undertaken by M.I.C. Evaluator Strips (Oxoid, Basingstoke, UK), according to the manufacturer's instructions, with results confirmed using Etest (BioMérieux, Marcy l'Etoile, France) at Public Health England, Colindale, UK and the WHO Collaborating Centre for Gonorrhoea and other STIs, Sweden. European Committee on Antimicrobial Susceptibility Testing resistance breakpoints were used [2]. The minimum inhibitory concentrations (MICs) of nine antimicrobials are given in Table 1and demonstrate high-level resistance to azithromycin (> $256 \mathrm{mg} / \mathrm{L}$ ) and resistance to ceftriaxone $(0.5 \mathrm{mg} / \mathrm{L})$, as well as tetracycline ( $32 \mathrm{mg} / \mathrm{L}$ ) and ciprofloxacin (> 32 $\mathrm{mg} / \mathrm{L}$ ). The patient was recalled and during this visit, 13 days after starting ceftriaxone/doxycycline treatment, his symptoms had resolved and a urine NAAT was negative for $N$. gonorrhoeae. However, given the previous antibiotic susceptibility profile, he was treated with a single dose of intramuscular spectinomycin $(2 \mathrm{~g})$. At a follow-up appointment, 20 days later, a urine NAAT was negative but a pharyngeal swab (omitted accidentally 
TABLE 1

Antimicrobial minimum inhibitory concentrations, Neisseria gonorrhoeae case imported from Thailand to England, February 2018

\begin{tabular}{|c|c|c|}
\hline Antimicrobial & MIC & Interpretation ${ }^{\mathrm{a}}$ \\
\hline Ceftriaxone & $0.5 \mathrm{mg} / \mathrm{L}$ & Resistant \\
\hline Cefixime & $2 \mathrm{mg} / \mathrm{L}$ & Resistant \\
\hline Azithromycin & $>256 \mathrm{mg} / \mathrm{L}$ & High-level resistant \\
\hline Ciprofloxacin & $>32 \mathrm{mg} / \mathrm{L}$ & Resistant \\
\hline Tetracycline & $32 \mathrm{mg} / \mathrm{L}$ & Resistant \\
\hline Benzylpenicillin & $1 \mathrm{mg} / \mathrm{L}$ & intermediate susceptible \\
\hline Spectinomycin & $8 \mathrm{mg} / \mathrm{L}$ & Susceptible \\
\hline Gentamicin & $2 \mathrm{mg} / \mathrm{L}$ & No resistance breakpoint available (low value) \\
\hline Ertapenem & $0.032 \mathrm{mg} / \mathrm{L}$ & No resistance breakpoint available (low value) \\
\hline
\end{tabular}

MIC: minimum inhibitory concentrations.

a European Committee on Antimicrobial Susceptibility Testing resistance breakpoints were used [2].

\section{TABLE 2}

Antimicrobial resistance determinants present in isolates, Neisseria gonorrhoeae case imported from Thailand to England, February 2018

\begin{tabular}{|c|c|c|c|}
\hline Gene & Variant & Mechanism & Antimicrobials affected \\
\hline $23 \mathrm{~S}$ rRNA & A2059G, 4 copies & Decreased macrolide binding to $50 \mathrm{~S}$ ribosome & AZM \\
\hline penA & FC428 mosaic penA - 100\% identity & $\begin{array}{c}\text { Reduced } \beta \text {-lactam acylation of penicillin binding } \\
\text { protein (PBP) } 2\end{array}$ & CRO, PEN \\
\hline penB & G120K, A121D & Reduced influx through PorB1b & CRO, PEN, TET \\
\hline$m t r R$ & G45D, Promoter deletion & $\begin{array}{l}\text { Over-expression of MtrCDE efflux pump resulting in } \\
\text { increased efflux }\end{array}$ & AZM, CRO, PEN, TET \\
\hline ponA & L421P & Reduced $\beta$-lactam acylation of $\mathrm{PBP}_{1}$ & PEN \\
\hline tetM & Gene presence & Prevents tetracycline binding to the $30 \mathrm{~S}$ ribosome & TET \\
\hline rps/ & $V_{57 M}$ & Reduced affinity of $30 \mathrm{~S}$ ribosome for tetracycline & TET \\
\hline gyrA & S91F, D95A & Reduced quinolone binding to DNA gyrase & CIP \\
\hline parC & S87R & Reduced quinolone binding to topoisomerase IV & CIP \\
\hline
\end{tabular}

AZM: azithromycin; CIP: ciprofloxacin; CRO: ceftriaxone; PEN: benzylpenicillin; TET: tetracycline.

Resistance determinants as previously described [5] were searched for. No additional macAB, norM promoter variants; $m$ trR, pilQ mutations; or erm, mef, ere genes were identified. The FC428 penA allele has NCBI accession number LC113953.1.

at the prior visits; the patient was asymptomatic at this site) was culture-positive for $N$. gonorrhoeae thus fulfilling international criteria for a verified treatment failure [3] as no further contact was reported with the Thai female following initial treatment. The same MICs were obtained from both cultured isolates from the case. Following this, the patient was treated with ertapenem ( $1 \mathrm{~g}$ ) intravenously for 3 days. Subsequent NAAT and culture of urethral and pharyngeal swabs a further 21 days later were negative. The patient's regular UK partner had negative vaginal and pharyngeal swab NAATs. We were unable to contact the patient's partner from Thailand who the patient had initially met via a dating website.

\section{Antimicrobial resistance determinants and sequence analysis}

By combining short-read (Illumina, San Diego, California, United States of America) and long-read (Oxford Nanopore Technologies, Oxford, UK) sequence data a complete hybrid $2.17 \mathrm{Mb}$ assembly for each isolate was determined, these have been deposited, together with raw sequence data, in the European Nucleotide Archive (PRJEB26560; see supplement for details). Mapping sequence reads to one of these novel reference genomes as previously described [4], the sequences of the pre-treatment urethral (G97687) and the post-initial treatment pharyngeal (G7944) isolates from the case were indistinguishable i.e. they were consistent with acquisition from the same source at both anatomical sites. The isolates had a novel N. gonorrhoeae multi-antigen sequence typing (NG-MAST) 


\section{FIGURE 1}

Genetic relatedness with previous ceftriaxone resistant isolates of Neisseria gonorrhoeae case imported from Thailand to England, February 2018

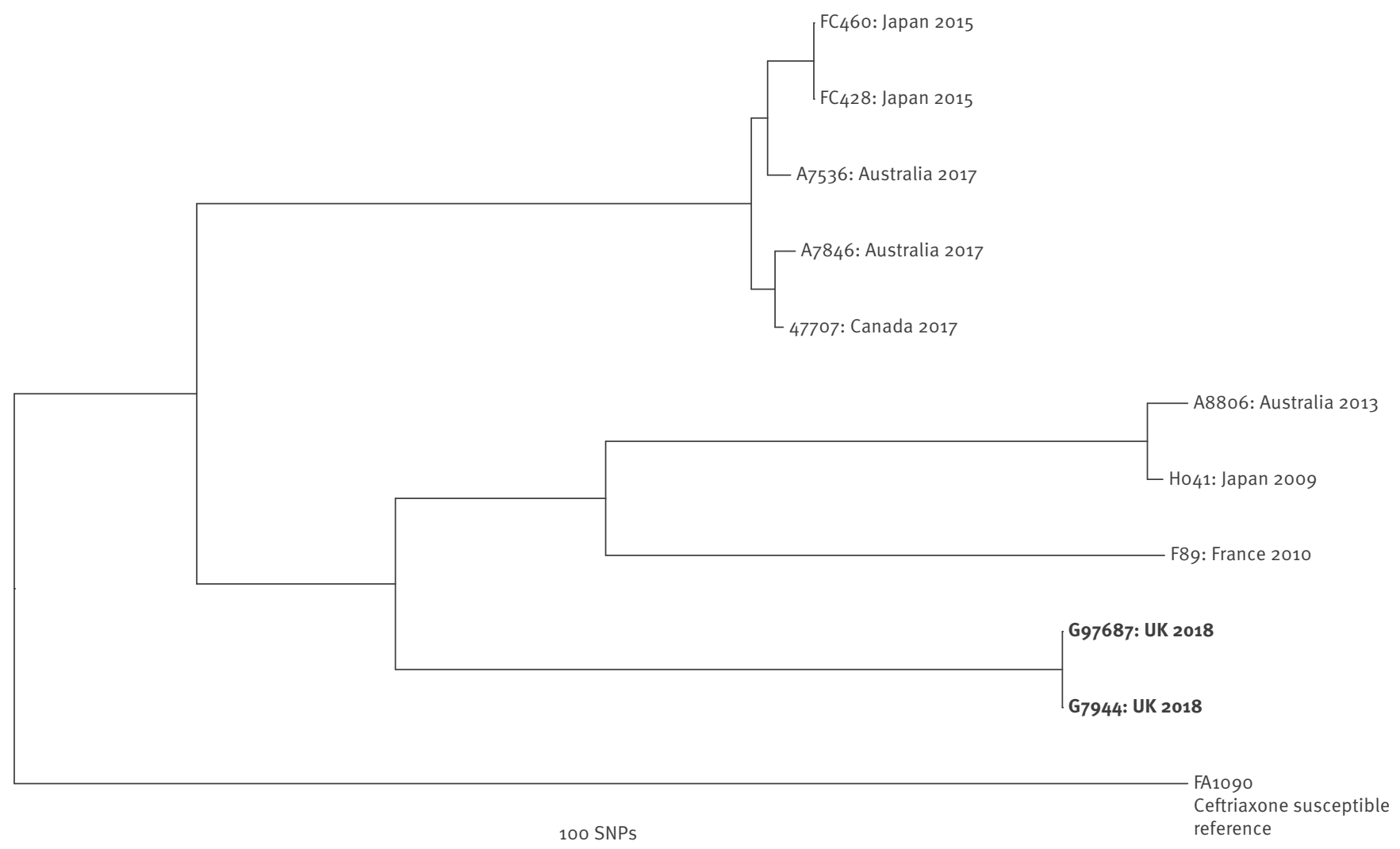

The figure shows a recombination-corrected maximum-likelihood phylogeny based on mapping to the Neisseria gonorrhoeae NCCP11945 reference genome. It shows the relationship between the case's isolates' sequences and previously sequenced N. gonorrhoeae ceftriaxone resistant isolates (from NCBI/ENA BioProjects PRJNA416507, PRJNA415047, PRJEB14020 and GenBank accession number NC_002946.2) [6,7,14$17,19,21]$. Isolates from the case are in bold and labelled with the identifiers used in the text.

sequence type (ST), ST16848 determined in silico [4], and were assigned MLST 12039.

Antimicrobial resistance determinants were identified from the sequencing data as described previously using a combination of de novo assembly and mapping-based approaches [5]. The isolates from the case included an identical mosaic penA allele to that previously identified in the ceftriaxone-resistant FC428 strain isolated in Japan in 2015, which also had a ceftriaxone MIC of $0.5 \mathrm{mg} / \mathrm{L}$ [6]. This mosaic penA allele contains two key ceftriaxone resistance mutations, $\mathrm{A} 311 \mathrm{~V}$ and $\mathrm{T} 483 \mathrm{~S}$, but not the T316P mutation found in the more resistant Ho41 strain [7]. The isolates also had the $\mathrm{A} 2059 \mathrm{G}$ mutation in all four $23 \mathrm{~S}$ rRNA genes - the most commonly occurring mutation responsible for high-level azithromycin resistance [8]. Additional antimicrobial resistance determinants are summarised in Table 2. The isolate had a N. gonorrhoeae Sequence Typing for Antimicrobial Resistance (NG-STAR) type of 996.

Based on reference-based mapping and correcting for recombination [4], previously sequenced ceftriaxone-resistant N. gonorrhoeae isolates, including the $\mathrm{FC}_{428}$ sharing the same penA allele, differed from our case by> 1,500 single nucleotide polymorphisms (SNPs) (Figure 1).

We compared our isolates' sequences to all publicly available whole-genome sequenced $N$. gonorrhoeae isolates. There were 7,812 sequences available for comparison, including European and North American surveillance samples and samples from a global collection. Using a rapid k-mer based search (see supplement for details), we identified the 98 most closely related genomes to the two sequences identified in our case to determine a maximum-likelihood phylogeny (Figure 2). The phylogeny contains two deep clades, the top clade contains the sequences from our case, which is predominantly from NG-MAST ST4995 and closely-related STs (collectively known as genogroup G4995); our case differs from G4995 by approximately 400 SNPs. The most closely related genomes were within 45 SNPs and were obtained in China (isolation dates unavailable) with additional genomes within 54 and 71 SNPs from the UK (2014) and Japan (2015), respectively. N. gonorrhoeae evolves at 3.6 SNPs per genome per year [4] meaning that the most recent 
Phylogeny of the most closely related existing Neisseria gonorrhoeae genome sequences to the imported Neisseria gonorrhoeae case from Thailand to England, February 2018

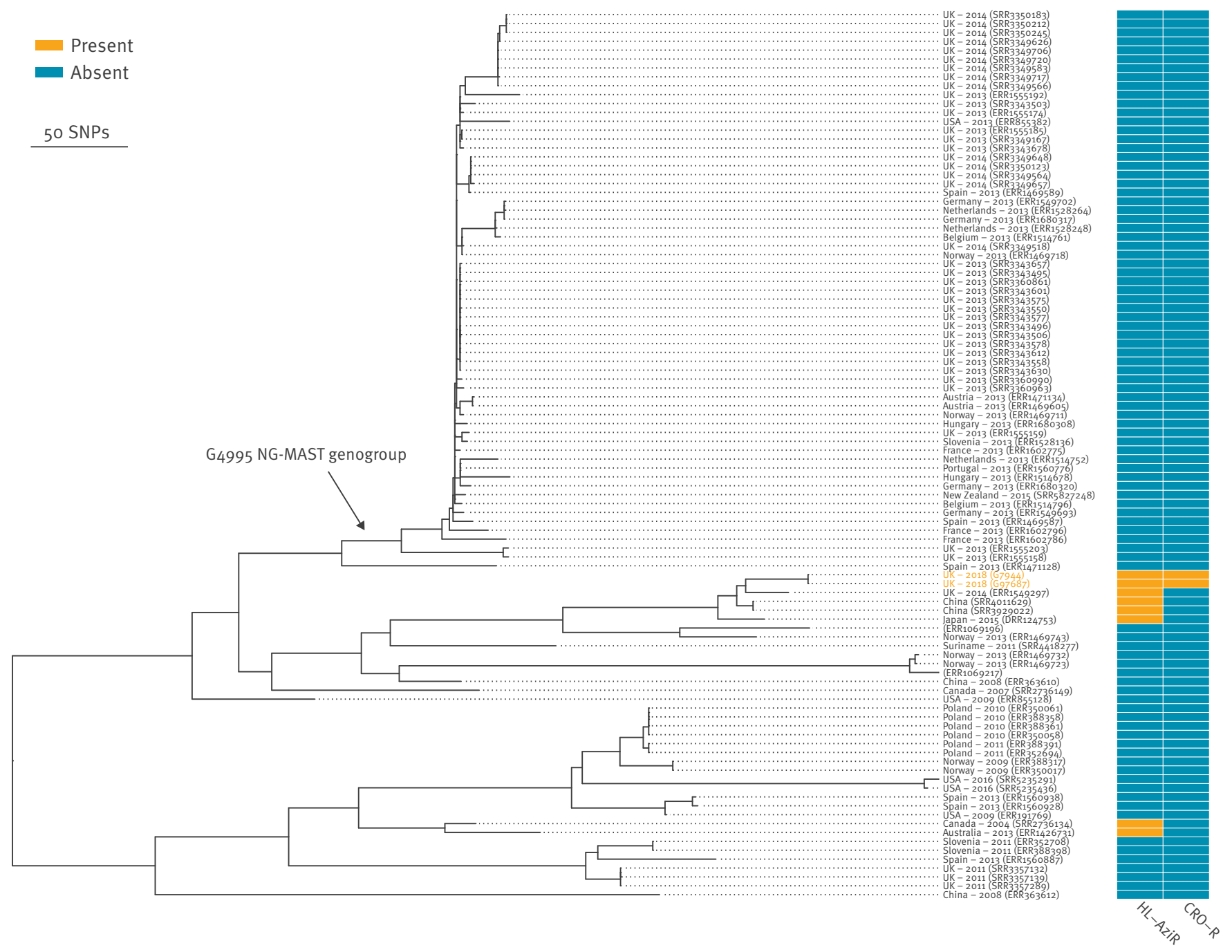

CRO-R: ceftriaxone resistant; HL-AziR: high-level azithromycin resistant.

The figure shows a recombination-corrected maximum-likelihood phylogeny based on mapping to a novel hybrid assembly of the case's sequences (ENA Sample ERS2461487). It shows the relationship between isolate sequences identified from our case and the most closely genetically related previously sequenced Neisseria gonorrhoeae isolates. Sequences are labelled with country and year of isolation where available (obtained either from the European Nucleotide Archive or the sequence submitter); sequence run accession numbers are provided. Antimicrobial susceptibilities were inferred from available sequence data [5]. Isolates from the case are shown in bold and labelled with the identifiers used in the text. The NG-MAST genogroup G4995's most recent common ancestor is indicated by an arrow.

common ancestor of these strains is likely to be from several years earlier. These related sequences each contained four copies of the $23 \mathrm{~S}$ rRNA A2059G mutation conferring high-level azithromycin resistance; however, all contained the ceftriaxone-susceptible penA II allele. No other sequence in the tree contained any of the three penA key mutations associated with ceftriaxone resistance i.e. A311V, T316P and T483S.

\section{Discussion}

This is the first reported ceftriaxone-resistant, highlevel azithromycin resistant $N$. gonorrhoeae isolate worldwide. The emergence of dual resistance to the last remaining mainstream treatment options poses serious challenges for the management and control of gonorrhoea infections globally. The strain was also resistant to ciprofloxacin and tetracycline. Although our case's urethral infection was cleared with empirical ceftriaxone/doxycycline treatment, his asymptomatic pharyngeal infection failed treatment despite the relatively high dose of ceftriaxone used ( $1 \mathrm{~g}$, compared to 250-500 mg frequently used) and the MIC just above the resistance breakpoint. This suggests that increased ceftriaxone dosing may not be adequate in similar cases. The case also failed spectinomycin treatment despite in vitro susceptibility. The limited efficacy of spectinomycin [9], as well as gentamicin [10,11] in pharyngeal infection, has been previously described. 
The first sustained national transmission of isolates with high-level resistance to azithromycin (MIC $\geq 256$ $\mathrm{mg} / \mathrm{L}$ ) has been recently documented in the UK [12], as well as a cluster of cases in Hawaii [13]. However, only a limited number of ceftriaxone-resistant isolates (MIC $\geq 0.25 \mathrm{mg} / \mathrm{L}$ ) cases have been characterised in detail worldwide. These include strains Ho41/WHO-X (Japan 2009, ceftriaxone MIC 2-4 mg/L) [7], F89/WHO-Y (France and Spain 2010, 1-2 mg/L) [14,15], A8806/ WHO-Z (Australia 2013, 0.5 mg/L) [16], GU140106 (Japan 2014, $0.5 \mathrm{mg} / \mathrm{L}$ ) [17], a strain in Argentina 2014 (0.5 $\mathrm{mg} / \mathrm{L}$ ) [18], and FC428 (Japan 2015, $0.5 \mathrm{mg} / \mathrm{L}$ ) [6]. While the earlier reports were not associated with apparent sustained onward transmission, subsequent spread of strains closely related to $\mathrm{FC}_{42} 2$ have been reported in Canada [19], Denmark [20] and Australia [21] in 2017 and are predominately associated with travel to southeast Asia. However, these previous strains were either susceptible, intermediate or had low-level resistance to azithromycin with MICs of $0.25^{-1} \mathrm{mg} / \mathrm{L}$. Six strains in a multi-institutional series from Japan (2000-2015) [22] and several isolates from a Chinese series (20132016) [23] were ceftriaxone-resistant with MICs of 0.5 $\mathrm{mg} / \mathrm{L}$, as well as a case from the United States in 2017 [24]. In 2014, combined azithromycin and ceftriaxone treatment failure was reported in the UK, with MICs of 1 and $0.25 \mathrm{mg} / \mathrm{L}$ respectively [25].

Dual resistance against recommended antibiotics for first-line treatment, including high-level azithromycin resistance, had not been reported until Public Health England issued an alert regarding this current case [26]. A similar public health alert has since been released by the Australian Department of Health, whereby they reported two further cases [27].

In our case, dual resistance arose via two known antimicrobial resistance mechanisms, which combined in the same isolate for the first recorded time. Comparing our isolates with all previously genome sequenced $N$. gonorrhoeaeisolates, we found that our isolates contain the same penA allele found in $\mathrm{FC}_{428}$ from Japan in 2015, but their overall genomes differ substantially (by $>1,500$ SNPS). The isolates from our case were more closely related (circa 400 SNPs) to those from genogroup $\mathrm{G} 4995$, which was the eighth most commonly circulating NG-MAST genogroup in Europe in 2013 [28]. To date, the spread of ceftriaxone-resistant strains has been limited, which is possibly due to the limited fitness of these strains. However, our isolate represents the concerning new combination of a ceftriaxone resistant penA allele that has disseminated to several countries including Japan, Australia, Canada, and Denmark, together with high-level azithromycin resistance, in a genetic background similar to a successful and commonly circulating strain.

\section{Conclusion}

Combined high-level resistance to azithromycin and resistance to ceftriaxone poses a global public health threat. Greater access to resistance testing, including through the development of new molecular diagnostics, is required to help guide treatment and ensure robust antimicrobial stewardship. This combined with increased use of test of cure, effective partner notification and fast-tracked development of new treatments and ideally a gonococcal vaccine, requires urgent action. The treatment failure also illustrates the severe difficulties in effectively treating pharyngeal gonorrhoea, and enhanced focus on appropriate detection and treatment of pharyngeal gonorrhoea, including in heterosexual men and women, is essential. This case, taken together with previous reports, highlights the global nature of antimicrobial resistance in gonorrhoea and in particular the importance of transmission in south-east Asia where systematic surveillance to date has been relatively limited. International cooperation is key to ensuring that gonorrhoea remains a treatable infection.

\section{Acknowledgements}

The research was supported by the National Institute for Health Research Health Protection Research Unit (NIHR HPRU) in Healthcare Associated Infections and Antimicrobial Resistance at the University of Oxford in partnership with Public Health England (PHE); and by the NIHR Biomedical Research Centre Oxford. The views expressed are those of the author(s) and not necessarily those of the NHS, the NIHR, the Department of Health or Public Health England. NIHR HPRU Independent Steering Committee: John Coia, Neil French, Charis Marwick, Mike Sharland. DWE is a NIHR Clinical Lecturer and Robertson Foundation Fellow. TEAP and DWC are NIHR Senior Investigators.

\section{Conflict of interest}

None declared.

Authors' contributions

DWE wrote the first draft of the manuscript. DWE, NDS and DG undertook the bioinformatic analysis. EL, NRR, AE clinically managed the case. KC and LB undertook the sequencing. MM, RN and MIA undertook the initial microbiological testing. GH, MJC, HF, DG and MU provided reference laboratory services and AE, MIA, GH, MJC, HF also formed part of a national response team. DWC and TEAP designed the sequencing experiments and provided advice on analysis and the manuscript. All authors reviewed and approved the final version of the manuscript.

\section{References}

1. Unemo M. Current and future antimicrobial treatment of gonorrhoea - the rapidly evolving Neisseria gonorrhoeae continues to challenge. BMC Infect Dis. 2015;15(1):364. https://doi.org/10.1186/s12879-015-1029-2 PMID: 26293005

2. The European Committee on Antimicrobial Susceptibility Testing (EUCAST). Breakpoint tables for interpretation of MICs and zone diameters. Version 8.1. Växjö: EUCAST; 2018. Available from: http://www.eucast.org.

3. Tapsall JW, Ndowa F, Lewis DA, Unemo M. Meeting the public health challenge of multidrug- and extensively drugresistant Neisseria gonorrhoeae. Expert Rev Anti Infect Ther. 2009;7(7):821-34. https://doi.org/10.1586/eri.09.63 PMID: 19735224 
4. De Silva D, Peters J, Cole K, Cole MJ, Cresswell F, Dean G, et al. Whole-genome sequencing to determine transmission of Neisseria gonorrhoeae: an observational study. Lancet Infect Dis. 2016;16(11):1295-303. https://doi.org/10.1016/S14733099(16)30157-8 PMID: 27427203

5. Eyre DW, De Silva D, Cole K, Peters J, Cole MJ, Grad YH, et al. WGS to predict antibiotic MICs for Neisseria gonorrhoeae. J Antimicrob Chemother. 2017;72(7):1937-47. https://doi. org/10.1093/jac/dkxo67 PMID: 28333355

6. Nakayama S, Shimuta K, Furubayashi K, Kawahata T, Unemo M, Ohnishi M. New Ceftriaxone- and Multidrug-Resistant Neisseria gonorrhoeae Strain with a Novel Mosaic penA Gene Isolated in Japan. Antimicrob Agents Chemother. 2016;60(7):4339-41. https://doi.org/10.1128/AAC.00504-16 PMID: 27067334

7. Ohnishi M, Golparian D, Shimuta K, Saika T, Hoshina S, Iwasaku K, et al. Is Neisseria gonorrhoeae initiating a future era of untreatable gonorrhea?: detailed characterization of the first strain with high-level resistance to ceftriaxone. Antimicrob Agents Chemother. 2011;55(7):3538-45. https://doi. org/10.1128/AAC.00325-11 PMID: 21576437

8. Unemo M, Shafer WM. Antimicrobial resistance in Neisseria gonorrhoeae in the 21st century: past, evolution, and future. Clin Microbiol Rev. 2014;27(3):587-613. https://doi. org/10.1128/CMR.00010-14 PMID: 24982323

9. Judson FN, Ehret JM, Handsfield HH. Comparative study of ceftriaxone and spectinomycin for treatment of pharyngeal and anorectal gonorrhea. JAMA. 1985;253(10):1417-9. https://doi. org/10.1001/jama.1985.03350340069019 PMID: 3155806

10. Dowell D, Kirkcaldy RD. Effectiveness of gentamicin for gonorrhoea treatment: systematic review and metaanalysis. Sex Transm Infect. 2012;88(8):589-94. https://doi. org/10.1136/sextrans-2012-050604 PMID: 22917693

11. Ross JD, Harding J, Duley L, Montgomery A, Hepburn T, Tan W, et al. LB1.5 The efficacy and safety of gentamicin for the treatment of genital, pharyngeal and rectal gonorrhoea: a randomised controlled trial. Sex Transm Infect. 2017;93(Suppl 2):A42-3.

12. Fifer H, Cole M, Hughes G, Padfield S, Smolarchuk C, Woodford $\mathrm{N}$, et al. Sustained transmission of high-level azithromycinresistant Neisseria gonorrhoeae in England: an observational study. Lancet Infect Dis. 2018;18(5):573-81. https://doi. org/10.1016/S1473-3099(18)30122-1 PMID: 29523496

13. Katz AR, Komeya AY, Kirkcaldy RD, Whelen AC, Soge 00, Papp JR, et al. Cluster of Neisseria gonorrhoeae Isolates With High-level Azithromycin Resistance and Decreased Ceftriaxone Susceptibility, Hawaii, 2016. Clin Infect Dis. 2017;65(6):918-23. https://doi.org/10.1093/cid/cix485 PMID: 28549097

14. Unemo M, Golparian D, Nicholas R, Ohnishi M, Gallay A, Sednaoui P. High-level cefixime- and ceftriaxone-resistant Neisseria gonorrhoeae in France: novel penA mosaic allele in a successful international clone causes treatment failure. Antimicrob Agents Chemother. 2012;56(3):1273-80. https:// doi.org/10.1128/AAC.05760-11 PMID: 22155830

15. Cámara J, Serra J, Ayats J, Bastida T, Carnicer-Pont D, Andreu A, et al. Molecular characterization of two high-level ceftriaxoneresistant Neisseria gonorrhoeae isolates detected in Catalonia, Spain. J Antimicrob Chemother. 2012;67(8):1858-60. https:// doi.org/10.1093/jac/dks162 PMID: 22566592

16. Lahra MM, Ryder N, Whiley DM. A new multidrug-resistant strain of Neisseria gonorrhoeae in Australia. N Engl J Med. 2014;371(19):1850-1. https://doi.org/10.1056/NEJMc1408109 PMID: 25372111

17. Deguchi T, Yasuda M, Hatazaki K, Kameyama K, Horie K, Kato T, et al. New Clinical Strain of Neisseria gonorrhoeae with Decreased Susceptibility to Ceftriaxone, Japan. Emerg Infect Dis. 2016;22(1):142-4. https://doi.org/10.3201/eid2201.150868 PMID: 26689442

18. Gianecini R, Oviedo C, Stafforini G, Galarza P. Neisseria gonorrhoeae Resistant to Ceftriaxone and Cefixime, Argentina. Emerg Infect Dis. 2016;22(6):1139-41. https://doi.org/10.3201/ eid2206.152091 PMID: 27191699

19. Lefebvre B, Martin I, Demczuk W, Deshaies L, Michaud S, Labbé AC, et al. Ceftriaxone-Resistant Neisseria gonorrhoeae, Canada, 2017. Emerg Infect Dis. 2018;24(2):381-3. https://doi. org/10.3201/eid2402.171756 PMID: 29131780

20. Terkelsen D, Tolstrup J, Johnsen $\mathrm{CH}$, Lund O, Larsen HK, Worning $P$, et al. Multidrug-resistant Neisseria gonorrhoeae infection with ceftriaxone resistance and intermediate resistance to azithromycin, Denmark, 2017. Euro Surveill. 2017;22(42):1273. https://doi.org/10.2807/1560-7917. ES.2017.22.42.17-00659 PMID: 29067905

21. Lahra MM, Martin I, Demczuk W, Jennison AV, Lee KI, Nakayama SI, et al. Cooperative Recognition of Internationally Disseminated Ceftriaxone-Resistant Neisseria gonorrhoeae Strain. Emerg Infect Dis. 2018;24(4):735-43. https://doi. org/10.3201/eid2404.171873 PMID: 29553335
22. Yasuda M, Hatazaki K, Ito S, Kitanohara M, Yoh M, Kojima M, et al. Antimicrobial Susceptibility of Neisseria gonorrhoeae in Japan from 2000 to 2015. Sex Transm Dis. 2017;44(3):149-53. PMID: 28178112

23. Yin Y-P, Han Y, Dai X-Q, Zheng HP, Chen SC, Zhu BY, et al. Susceptibility of Neisseria gonorrhoeae to azithromycin and ceftriaxone in China: A retrospective study of national surveillance data from 2013 to 2016. PLoS Med. 2018;15(2):e1002499. https://doi.org/10.1371/journal. pmed.1002499 PMID: 29408881

24. Abrams AJ, Kirkcaldy RD, Pettus K, Fox JL, Kubin G, Trees DL. A Case of Decreased Susceptibility to Ceftriaxone in Neisseria gonorrhoeae in the Absence of a Mosaic Penicillin-Binding Protein 2 (penA) Allele. Sex Transm Dis. 2017;44(8):492-4. https://doi.org/10.1097/OLQ.0000000000000645 PMID: 28703729

25. Fifer H, Natarajan U, Jones L, Alexander S, Hughes G, Golparian $D$, et al. Failure of Dual Antimicrobial Therapy in Treatment of Gonorrhea. N Engl J Med. 2016;374(25):2504-6. https://doi. org/10.1056/NEJMc1512757 PMID: 27332921

26. Public Health England. UK case of Neisseria gonorrhoeae with high-level resistance to azithromycin and resistance to ceftriaxone acquired abroad. 2018. Available from: https:// assets.publishing.service.gov.uk/government/uploads/ system/uploads/attachment_data/file/694655/hpr1118_ MDRGC.pdf

27. Australian Department of Health. Multi-drug resistant gonorrhoea in Australia. 2018. Available from: http://www. health.gov.au/internet/main/publishing.nsf/Content/ohpgonorrhoea.htm

28. Harris SR, Cole MJ, Spiteri G, Sánchez-Busó L, Golparian D, Jacobsson $\mathrm{S}$, et al. Euro-GASP study group. Public health surveillance of multidrug-resistant clones of Neisseria gonorrhoeae in Europe: a genomic survey. Lancet Infect Dis. 2018;18(7):758-68. https://doi.org/10.1016/S14733099(18)30225-1 PMID: 29776807

\section{License and copyright}

This is an open-access article distributed under the terms of the Creative Commons Attribution (CC BY 4.0) Licence. You may share and adapt the material, but must give appropriate credit to the source, provide a link to the licence, and indicate if changes were made.

This article is copyright of the authors or their affiliated institutions, 2018. 